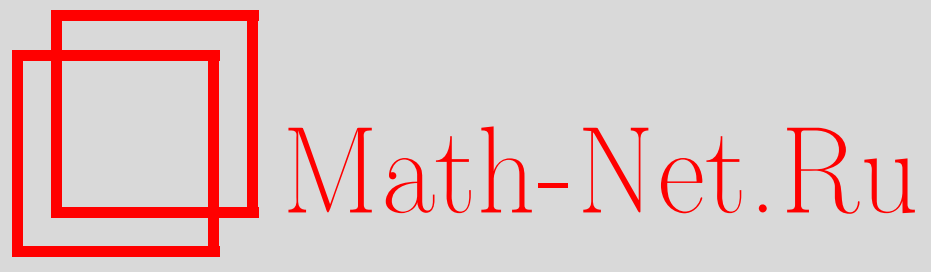

А. П. Комбаров, Счетная паракомпактность и нормальные функторы, Матем. заметки, 2015, том 98, выпуск $5,794-796$

DOI: https://doi.org/10.4213/mzm10899

Использование Общероссийского математического портала Math-Net.Ru подразумевает, что вы прочитали и согласны с пользовательским соглашением http://www . mathnet.ru/rus/agreement

Параметры загрузки:

IP: 54.80 .97 .219

26 апреля 2023 г., 12:02:38

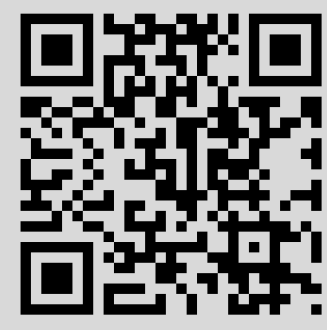




\section{Счетная паракомпактность и нормальные функторы}

\section{А. П. Комбаров}

Все топологические пространства в этой заметке предполагаются регулярными. Терминология и обозначения, не разъясняемые ниже, такие же, как в книге [1]. В статье [2] доказана следующая

Теорема 1. Пусть $X$ - паракомпактное р-пространство, $\mathscr{F}$ - нормальный функтор степени $\geqslant 3$, действующий в категории Я Раракомпактных р-пространств и их совершенных отображений. Тогда если пространство $\mathscr{F}(X)$ наследственно нормально, то $X$ метризуемое пространство.

Паракомпактные $p$-пространства - это в точности полные прообразы метрических пространств при совершенных отображениях [3]. Ковариантный функтор $\mathscr{F}$ в категории $\mathscr{P}$ называется нормальным, если функтор $\mathscr{F}$ непрерывен, мономорфен, эпиморфен, сохраняет вес, пересечения, прообразы, точку и пустое множество (см. [4]), а также удовлетворяет условию, взятому из работы [5]: для любого паракомпактного $p$-пространства $X$ и любого натурального $n$ отображение $\pi_{n}: X^{n} \times \mathscr{F}(n) \rightarrow \mathscr{F}(X)$ непрерывно. Отображение Басманова

$$
\pi_{n}: X^{n} \times \mathscr{F}(n) \rightarrow \mathscr{F}(X)
$$

определяется равенством

$$
\pi_{n}(\xi, a)=\mathscr{F}(\xi)(a),
$$

в котором каждая точка $\xi \in X^{n}$ отождествляется с отображением $\xi: n \rightarrow X$. Здесь $n$ обозначает дискретное пространство, состоящее из $n$ точек. Условия непрерывности, мономорфности, эпиморфности, сохранения веса, пересечения, прообразов, точки, пустого множества, входящие в определение нормального функтора в категории $\mathscr{P}$, аналогичны условиям из определения нормального функтора в категории Comp всех компактов и их непрерывных отображений. Поэтому ограничение нормального функтора в категории $\mathscr{P}$ на подкатегорию Сотр является нормальным функтором в категории Сотр в смысле определения, принадлежащего Щепину (см. [6], [4]); при этом теорема 1 переходит в известную теорему Федорчука [7], которая, тем самым, является следствием теоремы 1. Другое обобщение теоремы Федорчука содержится в работе [8]. Известно, что если $\mathscr{F}$ - нормальный функтор, то для любой точки $a \in \mathscr{F}(X)$ определен носитель $\operatorname{supp}(a)$ следующим образом:

$$
\operatorname{supp}(a)=\bigcap\{Y: Y \text { - замкнутое подмножество } X, a \in \mathscr{F}(Y)\} .
$$

Напомним также, что степень функтора $\operatorname{deg}(\mathscr{F})$ не превосходит числа $n$, если для любого $X$ и любой точки $a \in \mathscr{F}(X)$ справедливо неравенство $|\operatorname{supp}(a)| \leqslant n$.

Основным результатом настоящей заметки является теорема 2 , аналогичная теореме 1.

Теорема 2. Пусть $X$ - паракомпактное р-пространство, $\mathscr{F}$ - нормальный функтор степени $\geqslant 3$, действующий в категории Я Раракомпактных р-пространств и их совершенных отображений. Тогда если пространство $\mathscr{F}(X)$ наследственно счетно паракомпактно, то $X$ - метризуемое пространство.

Напомним, что пространство называется счетно паракомпактным, если в любое счетное открытое покрытие этого пространства можно вписать локально конечное открытое покрытие. Счетно паракомпактное пространство не обязано быть нормальным, и существуют примеры нормальных пространств, не являющихся счетно паракомпактными [1].

Теорема 2 является обобщением следующей теоремы 3, доказанной Жураевым [9].

DOI: $10.4213 / \mathrm{mzm} 10899$ 
Tеорема 3. Пусть $X$ - компакт, $\mathscr{F}$ - нормальный функтор степени $\geqslant 3$, действующий в категории Сотр. Тогда если пространство $\mathscr{F}(X)$ наследственно счетно паракомпактно, то X - метризуемое пространство.

Теорема Жураева в свою очередь усиливает теорему Зенора о метризуемости компакта, куб которого наследственно счетно паракомпактен [10]. Зенор [10] выводит свою теорему о кубе из следующей теоремы 4, которая необходима для доказательства теоремы 2.

Теорема 4. Если произведение $X \times Y$ наследственно счетно паракомпактно, то или $X$ совершенно нормально, или любое счетное дискретное подпространство пространства $Y$ замкнуто в $Y$.

Хорошо известно, что для произвольного хаусдорфова топологического пространства определено пространство $\exp _{\mathrm{c}}(X)$ компактных подмножеств пространства $X$ с топологией Вьеториса (см., например, [4]). Далее, для любого натурального числа $k$ через $\exp _{k}(X)$ обозначается подпространство пространства $\exp _{c}(X)$, состоящее из всех компактов, содержащих не более $k$ точек [4]. В статье [2] для любого совершенного отображения $f: X \rightarrow Y$ паракомпактного $p$-пространства $X$ на паракомпактное $p$-пространство $Y$ с помощью формулы $\left(\exp _{\mathrm{c}}(f)\right)(F)=f(F)$ определено отображение $\exp _{\mathrm{c}}(f): \exp _{\mathrm{c}}(X) \rightarrow \exp _{\mathrm{c}}(Y)$ и показано, что операция $\exp _{c}(\cdot)$ является нормальным функтором, действующим в категории $\mathscr{P}$, a $\exp _{k}(\cdot)$ является нормальным подфунктором функтора $\exp _{\mathrm{c}}(\cdot)$.

Следующая лемма 1 является следствием теоремы 3.

Лемма 1. Пусть $X$ - пространство точечно-счетного типа с единственной неизолированной точкой несчетного характера. Тогда $\exp _{3}(X)$ не является наследственно счетно паракомпактным пространством.

ДокАЗАтЕльство. В самом деле, в пространстве точечно-счетного типа любая точка содержится в компакте счетного характера. Если $C$ - компакт счетного характера, содержащий точку несчетного характера, то, очевидно, компакт $C$ не удовлетворяет первой аксиоме счетности в этой точке и, следовательно, не является метризуемым. Поскольку $\exp _{3}(\cdot)$ является нормальным функтором, по теореме 3 компакт $\exp _{3}(C)$ и, следовательно, $\exp _{3}(X)$ не являются наследственно счетно паракомпактными пространствами.

Лемма 2. Всякое паракомпактное $р$-пространство $X$ такое, что $\exp _{3}(X)$ наследственно счетно паракомпактное пространство, метризуемо.

ДокАЗАтЕльСтво. Если все точки пространства $X$ изолированы, то утверждение леммы 2, очевидно, выполняется. Заметим, что $X$ не может содержать единственную неизолированную точку, характер пространства в которой несчетен. Это следует из леммы 1 , поскольку любое паракомпактное $p$-пространство является пространством точечно-счетного типа. Если же $X$ содержит только одну неизолированную точку, характер пространства в которой счетен, то пространство $X$ обладает $\sigma$-дискретной базой и, следовательно, метризуемо $[1 ; 4.4 .8]$. Пусть теперь $X$ содержит две различные неизолированные точки $x_{1}$ и $x_{2}$. Выберем открытые множества $U_{1}$ и $U_{2}, V_{1}$ и $V_{2}$ так, чтобы

$$
x_{1} \in V_{1} \subset \bar{V}_{1} \subset U_{1}, \quad x_{2} \in V_{2} \subset \bar{V}_{2} \subset U_{2} \quad \text { и } \quad U_{1} \cap U_{2}=\varnothing .
$$

Очевидно, $\bar{V}_{1} \times \exp _{2}\left(\bar{V}_{2}\right)$ гомеоморфно подпространству $\exp _{3}(X)$, поскольку $\bar{V}_{1} \cap \bar{V}_{2}=\varnothing$. Множество $\bar{V}_{1}$ замкнуто в паракомпактном $p$-пространстве $X$, поэтому является паракомпактным $p$-пространством. Следовательно, $\bar{V}_{1}$ - пространство точечно-счетного типа с неизолированной точкой. Поэтому $\bar{V}_{1}$ содержит счетное дискретное незамкнутое подпространство. Тогда по теореме 4 пространство $\exp _{2}\left(\bar{V}_{2}\right)$ совершенно нормально. Из того, что $\bar{V}_{2}$ является паракомпактным $p$-пространством, следует, что и $\exp _{2}\left(\bar{V}_{2}\right)$ является паракомпактным $p$-пространством [2]. Из совершенной нормальности $\exp _{2}\left(\bar{V}_{2}\right)$ следует метризуемость $\bar{V}_{2}\left[2 ;\right.$ предложение 5]. Совершенно аналогично показывается, что и $\bar{V}_{1}$ метризуемо. Итак, доказано, что если паракомпактное $p$-пространство $X$ такое, что пространство 
$\exp _{3}(X)$ наследственно счетно паракомпактно, содержит по крайней мере две неизолированные точки, то у любой неизолированной точки пространства $X$ есть метризуемая окрестность. Приведенное рассуждение показывает, что паракомпактное $p$-пространство $X$ локально метризуемо и поэтому метризуемо [1]. Лемма 2 доказана.

Из утверждений 2.1.4 и 5.2.G книги [1] легко выводится следующая лемма 3.

Лемма 3. Образ наследственно счетно паракомпактного пространства при совершенном отображении является наследственно счетно паракомпактным пространством.

ДокАЗАТЕЛЬСТво теОремы 2. Пусть $\mathscr{F}-$ нормальный функтор степени $\geqslant 3$. Тогда в пространстве $\mathscr{F}(3)$ найдется элемент $a \in \mathscr{F}(3)$ такой, что $|\operatorname{supp}(a)|=3$. Определим $\mathscr{F}_{a}(X)=\pi_{3}\left(X^{3} \times\{a\}\right)$. Очевидно, $\mathscr{F}_{a}(X) \subset \mathscr{F}(X)$. Будем рассматривать отображение supp как однозначное, действующее из $\mathscr{F}_{a}(X)$ в $\exp _{3}(X)$. Известно [2; лемма 1], что отображение supp: $\mathscr{F}_{a}(X) \rightarrow \exp _{3}(X)$ совершенно и является эпиморфизмом. Наследственно счетно паракомпактное пространство $\mathscr{F}(X)$ содержит подпространство $\mathscr{F} a(X)$, поэтому пространство $\mathscr{F}_{a}(X)$ также наследственно счетно паракомпактно. По лемме 3 совершенный образ пространства $\mathscr{F}_{a}(X)$ также является наследственно счетно паракомпактным пространством. Следовательно, пространство $\exp _{3}(X)$ наследственно счетно паракомпактно. Применение леммы 2 завершает доказательство теоремы 2.

В заключение вопрос. Можно ли усилить теорему 2, заменив наследственную счетную паракомпактность на наследственную $\delta$-нормальность (см. [11], [12])?

\section{СПИСОК ЦИТИРОВАННОЙ ЛИТЕРАТУРЫ}

[1] Р. Энгелькинг, Общая топология, Мир, М., 1986. [2] М. А. Добрынина, Матем. заметки, 90:4 (2011), 630-633. [3] А. В. Архангельский, Матем. сб., 67:1 (1965), 55-88. [4] В.В. Федорчук, В. В. Филиппов, Общая топология. Основные конструкции, Изд-во Моск. ун-та, М., 1988. [5] В.Н. Басманов, Вестн. Моск. ун-та. Сер. 1. Матем., мех., 1984, № 6, 40-42. [6] Е. В. Щепин, УМН, 36:3 (1981), 3-62. [7] В. В. Федорчук, Вестн. Моск. ун-та. Сер. 1. Матем., мех., 1989, № 4, 93-96. [8] А. П. Комбаров, Матем. заметки, 76:1 (2004), 147-149. [9] Т. Ф. Жураев, Вестн. Моск. ун-та. Сер. 1. Матем., мех., 2000, № 4, 8-11. [10] P. Zenor, Proc. Amer. Math. Soc., 30 (1971), 199-201. [11] А. П. Комбаров, Матем. заметки, 92:5 (2012), 786-788. [12] А. П. Комбаров, Матем. заметки, 94:3 (2013), $473-476$.

\section{А. П. Комбаров}

Поступило

Московский государственный

03.04.2015

университет имени М.В. Ломоносова

E-mail: apkombarov@gmail.com 